\title{
Robustness with respect to small time-varied delay for linear dynamical systems on Banach spaces
}

\author{
by \\ Miao Li, Xiao-Hui Gu and Fa-Lun Huang (Chengdu)
}

\begin{abstract}
Under suitable conditions we prove the wellposedness of small time-varied delay equations and then establish the robust stability for such systems on the phase space of continuous vector-valued functions.
\end{abstract}

1. Introduction. The robustness of delay equations has been studied by many authors (see cf. [Ba1, Ba2, Da, EN, Hu, FN, JGH, Liu]).

In this paper we consider the time-varied delay equation of the form

$$
\begin{cases}x^{\prime}(t)=A x(t)+B x(t-\tau(t)), & t \geq 0, \\ x(\theta)=\xi(\theta), & -r \leq \theta \leq 0,\end{cases}
$$

where $A$ generates a $C_{0}$-semigroup $(T(t))_{t \geq 0}$ on a Banach space $X, B$ is a closed densely defined linear operator on $X, \tau(t)$ is continuous and $\xi$ is taken from some phase space.

Huang $([\mathrm{Hu}])$ proved the robust stability of the delay equation (1.1) on the phase space $C(-r, 0 ; X)$ in the case that $B$ is a bounded operator. Dropping the assumption that $B$ is bounded, Liu ([Liu]) showed that if $A$ generates a holomorphic semigroup and $B$ is $(-A)^{\alpha}$-bounded, then the exponential stability of (1.1) (with $\tau(t) \equiv r$ ) on the phase space $C(-r, 0 ; D(A)$ ) is robust. Bátkai et al. ([Ba1, Ba2]) proved a similar result on the phase space $X \times L^{p}(-r, 0 ; D(B))$.

Our goal in this paper is to study the robust stability of the time-varied delay equation (1.1) in the case that $B$ is unbounded. The organization of the paper is as follows: in Section 2, we will prove the wellposedness of (1.1) under some general assumptions on $A$ and $B$, and that the solution operators are given by Dyson-Phillips series. In Section 3, we prove the robust stability of the equation with time-varied delay on the phase space of continuous functions under the assumption that $B T(t)$ is norm continuous

2000 Mathematics Subject Classification: 34K05, 34K20, 34G10, 47D06.

Key words and phrases: $C_{0}$-semigroup, delay equations, robust stability.

This project is supported by Tianyuan Science Foundation of China (No. 10226030). 
for $t>0$. In addition, we will give an example to show that under this condition, the semigroup $T(t)$ is not necessarily holomorphic. So our results in this section generalize that of [Liu]. Moreover, our results show that on the phase space of continuous functions, the robust stability of the system without delay persists in the system with time-varied delay. However, the time-varied delay on the phase space $X \times L^{p}(-r, 0 ; D(B))$ will greatly affect the robustness and even the wellposedness of the delay equation. This will be taken up in a subsequent paper.

2. Preliminaries and wellposedness. Let $X$ be a Banach space with norm $\|\cdot\|$ and let $\mathbf{B}(X)$ be the Banach algebra of all bounded linear operators on $X$. If $A$ is a linear operator on $X$, we write $D(A)$ for its domain. We denote by $(f * g)(t)=\int_{0}^{t} f(t-s) g(s) d s$ the convolution of $f$ and $g$. Throughout this paper the following assumptions will be in force: on $X$.

General Assumptions. (a1) $A$ generates a $C_{0}$-semigroup $(T(t))_{t \geq 0}$

(a2) $B$ is a closed linear operator on $X, D(A) \subset D(B)$ and there is a non-negative measurable function $k \in L_{\mathrm{loc}}^{1}(0, \infty)$ such that

$$
\|B T(t) x\| \leq k(t)\|x\|, \quad t \geq 0, x \in D(A) .
$$

Since $k \in L_{\text {loc }}^{1}(0, \infty)$, from [DS, pp. 631, Theorem 19] one knows that $A+B$ with domain $D(A)$ generates a $C_{0}$-semigroup $\left(T_{B}(t)\right)_{t \geq 0}$ on $X$.

Let $\omega_{0}(T)$ be the growth bound of $(T(t))_{t \geq 0}$, that is, for $\omega>\omega_{0}(T)$ and $0<\delta<\omega-\omega_{0}(T)$, there is a constant $M \geq 1$ such that $\|T(t)\| \leq M e^{(\omega-\delta) t}$ for $t \geq 0$. Let $t_{0}>0$ by such that $k\left(t_{0}\right)$ is finite. Then by (2.1), for $t \geq t_{0}$ and $x \in D(A)$, we have

$$
\begin{aligned}
\|B T(t) x\| & =\left\|B T\left(t_{0}\right) T\left(t-t_{0}\right) x\right\| \\
& \leq k\left(t_{0}\right)\left\|T\left(t-t_{0}\right) x\right\| \leq k\left(t_{0}\right) M e^{(\omega-\delta)\left(t-t_{0}\right)}\|x\| .
\end{aligned}
$$

This shows that $B T(t)$ extends to a bounded operator on $X$ for $t \geq t_{0}$ since $D(A)$ is dense. We will also denote this extension by $B T(t)$ in the rest of this paper. Moreover, since there is a sequence $\left\{t_{n}\right\} \subset \mathbb{R}^{+}$such that $t_{n} \rightarrow 0$ and $k\left(t_{n}\right)$ is finite, we know that $B T(t) \in \mathbf{B}(X)$ for all $t>0$. Let $k_{0}(t)=\|B T(t)\|$. By $(2.2)$, we have $k^{0}(t):=k_{0}(t) e^{-\omega t} \in L^{1}(0, \infty)$ and

$$
k^{0}(t) \leq k_{0}\left(t_{0}\right) M e^{-\delta\left(t-t_{0}\right)}, \quad t \geq t_{0} .
$$

Furthermore, we have

Lemma 2.1. For all $t>0, B T_{B}(t) \in \mathbf{B}(X)$ and $k_{1}(t):=\left\|B T_{B}(t)\right\| \in$ $L_{\mathrm{loc}}^{1}(0, \infty)$ satisfies

$$
k_{1}(t) \leq k_{1}\left(t_{0}\right) M e^{(\omega-\delta)\left(t-t_{0}\right)}, \quad t \geq t_{0}>0,
$$


where $\omega>\max \left\{0, \omega_{0}(T), \omega_{0}\left(T_{B}\right)\right\}$ is large enough such that $\|T(t)\|,\left\|T_{B}(t)\right\|$ $\leq M e^{(\omega-\delta) t}$ for $t \geq 0$ and some constant $M \geq 1$, and

$$
\beta:=M \int_{0}^{\infty} k^{0}(t) d t<1 .
$$

Proof. Choose $\omega>\max \left\{0, \omega_{0}(T), \omega_{0}\left(T_{B}\right)\right\}$ large enough such that (2.5) holds. Then for $x \in X$ and $t>0$, multiplying the equation

$$
B T_{B}(t) x=B T(t) x+\int_{0}^{t} B T(t-s) B T_{B}(s) x d s
$$

by $e^{-\omega t}$ yields

$$
e^{-\omega s}\left\|B T_{B}(s) x\right\| \leq k^{0}(s)\|x\|+\int_{0}^{s} k^{0}(s-\tau) e^{-\omega \tau}\left\|B T_{B}(\tau) x\right\| d \tau, \quad s>0
$$

Integrating (2.7) from 0 to $t$ gives

$$
\begin{aligned}
\int_{0}^{t} e^{-\omega s}\left\|B T_{B}(s) x\right\| d s & \leq \int_{0}^{t} k^{0}(s)\|x\| d s+\int_{00}^{t} \int_{0}^{s} k^{0}(s-\tau) e^{-\omega \tau}\left\|B T_{B}(\tau) x\right\| d \tau d s \\
& \leq \beta\|x\|+\int_{0}^{t} e^{-\omega \tau}\left\|B T_{B}(\tau) x\right\| \int_{\tau}^{t} k^{0}(s-\tau) d s d \tau \\
& \leq \beta\|x\|+\beta \int_{0}^{t} e^{-\omega \tau}\left\|B T_{B}(\tau) x\right\| d \tau .
\end{aligned}
$$

It follows that

$$
\int_{0}^{t} e^{-\omega s}\left\|B T_{B}(s) x\right\| d s \leq \beta(1-\beta)^{-1}\|x\|, \quad t>0, x \in X .
$$

By induction, from (2.7) using (2.8) we have

$$
e^{-\omega t}\left\|B T_{B}(t) x\right\| \leq k_{2}(t)\|x\|, \quad t>0, x \in X
$$

where $k_{2}(t)=\sum\left(k^{0}\right)^{* n}(t)$ and $\left(k^{0}\right)^{* n}=k^{0} * \cdots * k^{0}$ is the $n$-fold convolution of the kernel $k^{0}$. Since $\left\|k^{0}\right\|_{L^{1}(0, \infty)} \leq \beta$, we have $\left\|k_{2}\right\|_{L^{1}(0, \infty)} \leq(1-\beta)^{-1} \beta$. Thus from (2.9) we have $\left\|B T_{B}(t)\right\| \leq e^{\omega t} \beta(1-\beta)^{-1}$, and similarly to the proof of (2.2) and (2.3), one can show that (2.4) holds.

Next we consider the norm continuity of $B T(t)$ and $B T_{B}(t)$.

Lemma 2.2. If $B T(t)$ is norm continuous for $t>0$, then so is $B T_{B}(t)$. 
Proof. Let $t>0$ and $0<\delta<t / 2$. By (2.6), for $|h|<\delta$ and $x \in X$ satisfying $\|x\| \leq 1$,

$$
\begin{aligned}
\left\|B T_{B}(t+h) x-B T_{B}(t) x\right\| \\
=\| B(T(t+h) x-T(t)) x+\int_{t-\delta}^{t+h} B T(t+h-s) B T_{B}(s) x d s \\
\quad+\int_{0}^{t-\delta}[B T(t+h-s)-B T(t-s)] B T_{B}(s) x d s \\
\quad-\int_{t-\delta}^{t} B T(t-s) B T_{B}(s) x d s \| \\
\leq\|B T(t+h)-B T(t)\|+\int_{0}^{t-\delta}\|B T(t+h-s)-B T(t-s)\| k_{1}(s) d s \\
\quad+M_{t}\left[\int_{t-\delta}^{t+h} k_{0}(t+h-s) d s+\int_{t-\delta}^{t} k_{0}(t-s) d s\right],
\end{aligned}
$$

where $M_{t}:=\max \left\{k_{1}(s): t / 2 \leq s \leq 3 t / 2\right\} ;(2.4)$ implies that $M_{t}$ is finite for $t>0$. Since $B T(t)$ is norm continuous for $t>0$ and $k_{0} \in L_{\text {loc }}^{1}(0, \infty)$, for every $\varepsilon>0$ there is a $\delta_{1} \in(0, t / 4)$ such that when $|h|<\delta / 2$ and $\delta \leq \delta_{1}$,

$$
\|B T(t+h)-B T(t)\|+M_{t}\left(\int_{t-\delta}^{t+h} k_{0}(t+h-s) d s+\int_{t-\delta}^{t} k_{0}(t-s) d s\right)<\varepsilon / 2 .
$$

Moreover, for given $0<\delta \leq \delta_{1}$, since $B T(t)$ is uniformly continuous on $[\delta / 2, t+\delta / 2]$, there exists $\delta_{\varepsilon} \in(0, \delta / 2)$ such that for $s \in[0, t-\delta]$ and $|h| \leq \delta_{\varepsilon}$

$$
\|B T(t+h-s)-B T(t-s)\|<\frac{1}{2}\left(\int_{0}^{t} k_{1}(s) d s\right)^{-1} \varepsilon .
$$

Combining all these inequalities, for $x \in X$ with $\|x\| \leq 1$ and $|h|<\delta_{\varepsilon}$ we obtain

$$
\left\|B T_{B}(t+h) x-B T_{B}(t) x\right\|<\varepsilon,
$$

which implies the norm continuity of $B T_{B}(t)$ on $(0, \infty)$.

The continuity of $B T(t) x$ for some point $x$ is equivalent to that of $B T_{B}(t) x$ :

Lemma 2.3. Let $x \in D(B)$. Then $B T(t) x$ is continuous for $t \geq 0$ if and only if $B T_{B}(t) x$ is continuous for $t \geq 0$. 
Proof. Suppose that $B T(t) x$ is continuous for $t \geq 0$. Since for $0 \leq t \leq 1$,

$$
\begin{aligned}
\left\|B T_{B}(t) x-B x\right\| & =\left\|B T(t) x-B x+\int_{0}^{t} B T_{B}(t-s) B T(s) x d s\right\| \\
& \leq\|B T(t) x-B x\|+\int_{0}^{t} k_{1}(t-s) d s \max _{0 \leq \tau \leq 1}\|B T(\tau) x\|,
\end{aligned}
$$

$B T_{B}(t) x$ is right-continuous at 0 . Now let $t>0$ and $|h|<\delta<\min \{1, t / 2\}$. Then

$$
\begin{aligned}
\left\|B T_{B}(t+h) x-B T_{B}(t) x\right\| & \| B T(t+h) x-B T(t) x+\int_{0}^{t+h} B T_{B}(s) B(t+h-s) x d s \\
= & \quad-\int_{0}^{t} B T_{B}(s) B T(t-s) x d s \| \\
\leq & \|B T(t+h) x-B T(t) x\| \\
& +\int_{0}^{t-\delta} k_{1}(s)\|B T(t+h-s) x-B T(t-s) x\| d s \\
& +\left[\int_{t-\delta}^{t+h} k_{1}(s) d s+\int_{t-\delta}^{t} k_{1}(s) d s\right] \max _{0 \leq \tau \leq 2}\|B T(\tau) x\| .
\end{aligned}
$$

Since $B T(s) x$ is uniformly continuous for $s \in[0, t+1]$ and $k_{1} \in L_{\mathrm{loc}}^{1}(0, \infty)$, for every $\varepsilon>0$ one can find a constant $\delta_{\varepsilon} \in(0, \min \{1, t / 2\})$ such that for $|h|<\delta<\delta_{\varepsilon}$

$$
\|B T(s+h) x-B T(s) x\|<\frac{1}{2}\left(1+\int_{0}^{t} k_{1}(s) d s\right)^{-1} \varepsilon, \quad s \in[0, t+1],
$$

and

$$
\int_{t-\delta}^{t+h} k_{1}(s) d s+\int_{t-\delta}^{t} k_{1}(s) d s<\frac{1}{2}\left(\max _{0 \leq \tau \leq 2}\|B T(\tau) x\|\right)^{-1} \varepsilon .
$$

By the above estimates, we have

$$
\left\|B T_{B}(t+h) x-B T_{B}(t) x\right\|<\varepsilon, \quad|h|<\delta_{\varepsilon},
$$

which means that $B T_{B}(t) x$ is continuous for $t \geq 0$. Conversely, if $B T_{B}(t) x$ is continuous for $t \geq 0$, then from

$$
B T(t) x=B T_{B}(t) x-\int_{0}^{t} B T(s) B T_{B}(t-s) x d s, \quad t \geq 0,
$$

by a similar argument one can show that $B T(t) x$ is continuous for $t \geq 0$. 
We are particularly interested in the subspace of $X$ on which $B T(t)$ (and also $B T_{B}(t)$ by Lemma 2.3$)$ is strongly continuous.

Lemma 2.4. Let $X_{b}$ be the subspace of $X$ defined by

$$
X_{b}=\{x \in D(B): B T(t) x \text { is continuous for } t \geq 0\} .
$$

Then $D(A) \subset X_{b} \subset D(B)$ and $X_{b}$ is a Banach space with norm

$$
\|x\|_{b}=\|x\|+\sup _{s \geq 0}\left\|e^{-\omega s} B T(s) x\right\|, \quad x \in X_{b},
$$

where $\omega>\max \left\{0, \omega_{0}(T), \omega_{0}\left(T_{B}\right)\right\}$ is large enough such that $\|T(t)\|+\left\|T_{B}(t)\right\|$ $\leq M e^{(\omega-\delta) t}$ for $t \geq 0$ and some constant $M \geq 1$, and

$$
\gamma:=M \int_{0}^{\infty} e^{-\omega t}\left(k_{0}(t)+k_{1}(t)\right) d t<1 .
$$

Moreover, the norm

$$
\|x\|_{b^{\prime}}:=\|x\|+\sup _{s \geq 0}\left\|e^{-\omega s} B T_{B}(s) x\right\|
$$

on $X_{b}$ is equivalent to $\|\cdot\|_{b}$. Finally, if $T_{B}(t)$ is exponentially stable, that is, there are constants $M_{b} \geq 1$ and $\omega_{b}>0$ such that $\left\|T_{B}(t)\right\| \leq M_{b} e^{-\omega_{b} t}$ for $t \geq 0$, then the norm

$$
\|x\|_{s}:=\|x\|+\sup _{s \geq 0}\left\|B T_{B}(s) x\right\|
$$

on $X_{b}$ is also equivalent to $\|\cdot\|_{b}$.

Proof. If $x \in D(A)$, then for $t \geq 0$ and $h>0$,

$$
\|B T(t+h) x-B T(t) x\|=\left\|B \int_{t}^{t+h} T(s) A x d s\right\| \leq \int_{t}^{t+h} k_{0}(s) d s \cdot\|A x\|,
$$

so $B T(t) x$ is continuous for $t \geq 0$ since $k_{0}(\cdot) \in L_{\text {loc }}^{1}(0, \infty)$. Hence $D(A) \subset$ $X_{b} \subset D(B)$.

Next we show that $\left(X_{b},\|\cdot\|_{b}\right)$ is a Banach space. Let $\left\{x_{n}\right\} \subset X_{b}$ be a Cauchy sequence in $X_{b}$. Then from the definition of the norm, both $\left\{x_{n}\right\}$ and $\left\{B x_{n}\right\}$ are Cauchy sequences in $X$ and thus converge. Suppose that $x_{n} \rightarrow x$ and $B x_{n} \rightarrow y$ in $X$. Then from the closedness of $B$ we have $x \in D(B)$ and $B x=y$. Now the strong continuity of $B T(t) x$ follows from the facts that $x_{n}$ converges to $x$ and the convergence of $B T(t) x_{n}$ to $B T(t) x$ is uniform in compact intervals. Similarly one can show that $\left(X_{b},\|\cdot\|_{b^{\prime}}\right)$ is also a Banach space by using Lemma 2.3 .

To see the equivalence of the two norms, by the Inverse Mapping Theorem, we only need to show that one norm is stronger than the other. Let $x \in X_{b}$. By the definition of $b^{\prime}$-norm we have

$$
e^{-\omega t}\left\|B T_{B}(t) x\right\| \leq\|x\|_{b^{\prime}}, \quad t \geq 0
$$


thus

$$
\begin{aligned}
&\|x\|_{b^{\prime}}=\|x\|+\sup _{s \geq 0}\left\|e^{-\omega s} B T_{B}(s) x\right\| \\
& \leq\|x\|+\sup _{s \geq 0}\left\|e^{-\omega s} B T(s) x\right\| \\
& \quad+\sup _{s \geq 0}\left\|\int_{0}^{s} e^{-\omega(s-\tau)} B T(s-\tau) e^{-\omega \tau} B T_{B}(\tau) x d \tau\right\| \\
& \leq\|x\|_{b}+\sup _{s \geq 0} \int_{0}^{s} e^{-\omega(s-\tau)} k_{0}(s-\tau) e^{-\omega \tau}\left\|B T_{B}(\tau) x\right\| d \tau \\
& \leq\|x\|_{b}+\sup _{s \geq 0} \int_{0} e^{-\omega(s-\tau)} k_{0}(s-\tau)\|x\|_{b^{\prime}} d \tau \\
& \leq\|x\|_{b}+\gamma\|x\|_{b^{\prime}} .
\end{aligned}
$$

It follows that $\|x\|_{b^{\prime}} \leq(1-\gamma)^{-1}\|x\|_{b}$ for $x \in X_{b}$, and therefore, the $\|\cdot\|_{b^{-n o r m}}$ is stronger than the $\|\cdot\|_{b^{\prime}}$-norm.

If $T_{B}(t)$ is exponentially stable, then by Lemma $2.1, B T_{B}(t) \in \mathbf{B}(X)$ for all $t>0$ and

$$
\left\|B T_{B}(t)\right\|=\left\|B T_{B}\left(t_{0}\right) T_{B}\left(t-t_{0}\right)\right\| \leq k_{1}\left(t_{0}\right) M_{b} e^{-\omega_{b}\left(t-t_{0}\right)}, \quad t \geq t_{0} .
$$

So $\|\cdot\|_{s}$ is a norm on $X_{b}$ and $\left(X_{b},\|\cdot\|_{s}\right)$ is a Banach space. Moreover, for $x \in X_{b}$,

$$
\|x\|_{b^{\prime}}=\|x\|+\sup _{s \geq 0}\left\|e^{-\omega s} B T_{B}(s) x\right\| \leq\|x\|+\sup _{s \geq 0}\left\|B T_{B}(s) x\right\|=\|x\|_{s},
$$

and again by the Inverse Mapping Theorem, the norms $\|\cdot\|_{s}$ and $\|\cdot\|_{b}$ on $X_{b}$ are equivalent.

After these preparations, we now consider the delay equation

$$
\begin{cases}x^{\prime}(t)=A x(t)+B x(t-\tau(t)), & t \geq 0, \\ x(\theta)=\xi(\theta), & -r \leq \theta \leq 0,\end{cases}
$$

where $0 \leq \tau \leq r, \tau(t)$ is continuous for $t \geq 0$ and $\xi(\cdot) \in C\left(-r, 0 ; X_{b}\right)$. In the rest of this paper we will denote by $\mathcal{X}=C\left(-r, 0 ; X_{b}\right)$ the phase space. The solution of (2.12) also satisfies

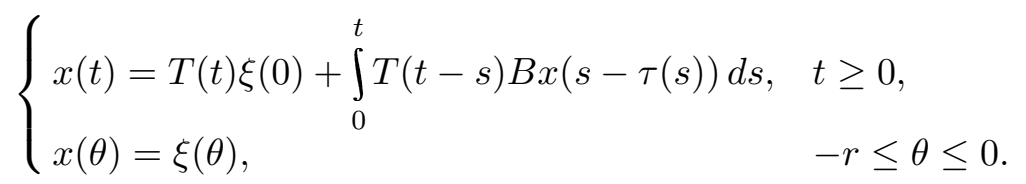

We call $x(t)$ a solution of (2.13) if $x(t) \in C\left(-r, \infty ; X_{b}\right)$ satisfies (2.13) and $x_{t}(\cdot) \in \mathcal{X}$ is continuous for $t \geq 0$, where $x_{t}(\theta):=x(t+\theta)$ for $t \geq 0$ and $-r \leq \theta \leq 0$. In the following we will denote the solution of (2.13) at $\xi$ by $x(t, \xi)$ and call it the mild solution of (2.12). 
TheOREM 2.5. For any $r>0$ and $\xi \in \mathcal{X},(2.13)$ has a unique solution $x(t, \xi)$. Let

$$
\left(T_{r}(t) \xi\right)(\theta):=x_{t}(\theta, \xi), \quad t \geq 0,-r \leq \theta \leq 0,
$$

be the solution operator. Then there exist positive constants $M_{0}$ and $\omega_{0}$, independent of $r$, such that

$$
\left\|T_{r}(t) \xi\right\|_{\mathcal{X}} \leq M_{0} e^{\omega_{0} t}\|\xi\|_{\mathcal{X}}, \quad t \geq 0, r>0, \xi \in \mathcal{X} .
$$

Proof. We will choose the $\|\cdot\|_{b}$-norm on $X_{b}$ given by (2.10), with the constant $\omega$ so large that $\|T(t)\| \leq M e^{(\omega-\delta) t}$ for all $t \geq 0$, and $\omega>\delta>M$ such that

$$
\beta_{0}:=M \int_{0}^{\infty} e^{-\omega t} k_{0}(t) d t<1-M \delta^{-1}
$$

For $r>0$ and $\xi \in \mathcal{X}$, define

$$
x^{(0)}(t)= \begin{cases}T(t) \xi(0), & t \geq 0, \\ \xi(t), & -r \leq t<0,\end{cases}
$$

and for $n=1,2, \ldots$,

$$
x^{(n)}(t)= \begin{cases}\int_{0}^{t} T(t-s) B x^{(n-1)}(s-\tau(s)) d s, & t \geq 0, \\ 0, & -r \leq t<0 .\end{cases}
$$

It is clear from the definition of $X_{b}$ that $x^{(0)}(t)$ is continuous for $t \geq-r$ in $X_{b}$, and from

$$
x_{t}^{(0)}(\theta)= \begin{cases}T(t+\theta) \xi(0), & t \geq r,-r \leq \theta \leq 0 \text { or } 0 \leq t \leq r,-t \leq \theta \leq 0, \\ \xi(t+\theta), & 0 \leq t \leq r,-r \leq \theta \leq-t,\end{cases}
$$

we have for $t \geq r,-r \leq \theta \leq 0$ or $0 \leq t \leq r,-t \leq \theta \leq 0$,

$$
\begin{aligned}
\left\|x_{t}^{(0)}(\theta)\right\|_{b} & =\|T(t+\theta) \xi(0)\|+\sup _{s \geq 0}\left\|e^{-\omega s} B T(s+t+\theta) \xi(0)\right\| \\
& \leq M e^{\omega(t+\theta)}\|\xi(0)\|+e^{\omega(t+\theta)} \sup _{s \geq 0}\left\|e^{-\omega(s+t+\theta)} B T(s+t+\theta) \xi(0)\right\| \\
& \leq M e^{\omega t}\|\xi(0)\|_{b}+e^{\omega t}\|\xi(0)\|_{b} \leq(1+M) e^{\omega t}\|\xi\|_{\mathcal{X}}
\end{aligned}
$$

and for $0 \leq t \leq r,-r \leq \theta \leq-t$,

$$
\left\|x_{t}^{(0)}(\theta)\right\|_{b}=\|\xi(t+\theta)\|_{b} \leq\|\xi\|_{\mathcal{X}}
$$

It follows that

$$
\left\|x_{t}^{(0)}(\cdot)\right\|_{\mathcal{X}} \leq(1+M) e^{\omega t}\|\xi\|_{\mathcal{X}}, \quad t \geq 0 .
$$

Moreover, from (2.15) it is easy to see that $x^{(1)}(t)$ is continuous for $t \geq 0$ in $X_{b}$ and by using (2.16) one can show that

$$
\left\|x_{t}^{(1)}(\cdot)\right\|_{\mathcal{X}} \leq(1+M) \beta_{1} e^{\omega t}\|\xi\|_{\mathcal{X}}, \quad t \geq 0, \xi \in \mathcal{X}
$$


where $\beta_{1}:=M \delta^{-1}+\beta_{0}<1$. Then by induction on $n$ we find that $x^{(n)}(t)$ is continuous in $X_{b}$ and

$$
\left\|x_{t}^{(n)}(\cdot)\right\|_{\mathcal{X}} \leq(1+M) \beta_{1}^{n} e^{\omega t}\|\xi\|_{\mathcal{X}}, \quad t \geq 0, n=0,1,2, \ldots
$$

Set $x(t)=\sum_{n=0}^{\infty} x^{(n)}(t)$ for $t \geq-r$. By $(2.17)$ the series $\sum_{n=0}^{\infty} x^{(n)}(t)$ is absolutely convergent on compact intervals in $X_{b}$ and

$$
\begin{aligned}
\left\|x_{t}(\cdot)\right\|_{\mathcal{X}} & \leq \sum_{n=0}^{\infty}\left\|x_{t}^{(n)}(\cdot)\right\|_{\mathcal{X}} \leq \sum_{n=0}^{\infty}(1+M) \beta_{1}^{n} e^{\omega t}\|\xi\|_{\mathcal{X}} \\
& =(1+M)\left(1-\beta_{1}\right)^{-1} e^{\omega t}\|\xi\|_{\mathcal{X}} .
\end{aligned}
$$

Thus $x(t)$ is continuous for $t \geq-r$ in $X_{b}$ and

$$
\begin{aligned}
x(t) & = \begin{cases}T(t) \xi(0)+\sum_{n=0}^{\infty} \int_{0}^{t} T(t-s) B x^{(n)}(s-\tau(s)) d s, & t \geq 0, \\
\xi(t), & -r \leq t \leq 0,\end{cases} \\
& = \begin{cases}T(t) \xi(0)+\int_{0}^{t} T(t-s) B \sum_{n=0}^{\infty} x^{(n)}(s-\tau(s)) d s, & t \geq 0, \\
\xi(t), & -r \leq t \leq 0,\end{cases} \\
& = \begin{cases}T(t) \xi(0)+\int_{0}^{t} T(t-s) B x(s-\tau(s)) d s, & t \geq 0, \\
\xi(t), & -r \leq t \leq 0,\end{cases}
\end{aligned}
$$

that is, $x(t)$ satisfies $(2.13)$ and by $(2.18)$,

$$
\left\|x_{t}(\cdot)\right\|_{\mathcal{X}} \leq(1+M)\left(1-\beta_{1}\right)^{-1} e^{\omega t}\|\xi\|_{\mathcal{X}}, \quad t \geq 0, \xi \in \mathcal{X} .
$$

To show the uniqueness of the solutions, let $x(t)$ be a solution of (2.13) with initial value $\xi(t) \equiv 0(t \in[-r, 0])$. Then $x(t)=0$ for $-r \leq t \leq 0$, while for $t \geq 0$,

$$
x(t)=\int_{0}^{t} T(t-s) B x(s-\tau(s)) d s .
$$

It is easy to show that for $t \geq r,-r \leq \theta \leq 0$ or $0 \leq t \leq r,-t \leq \theta \leq 0$,

$$
\left\|x_{t}(\theta)\right\|_{b} \leq M \int_{0}^{t+\theta}\left[e^{(\omega-\delta)(t+\theta-s)}+k_{0}(t+\theta-s)\right]\|x(s-\tau(s))\|_{b} d s,
$$

which implies that

$$
\left\|x_{t}(\cdot)\right\|_{\mathcal{X}} \leq \beta_{1} e^{\omega t}\left\|x_{t}(\cdot)\right\|_{\mathcal{X}}
$$

by using this inequality on the right-hand side of (2.20) and by induction one obtains

$$
\left\|x_{t}(\cdot)\right\|_{\mathcal{X}} \leq \beta_{1}^{n} e^{\omega t}\left\|x_{t}(\cdot)\right\|_{\mathcal{X}}, \quad n=1,2, \ldots
$$


Since $n$ is arbitrary and $\beta_{1}<1$, we have $x_{t} \equiv 0$, which proves the uniqueness of the solutions. So we can define

$$
\left(T_{r}(t) \xi\right)(\theta)=x(t+\theta, \xi), \quad t \geq 0,-r \leq \theta \leq 0, \xi \in \mathcal{X},
$$

where $x(t, \xi)$ is the solution of (2.13) at $\xi \in \mathcal{X}$. Moreover, (2.19) implies that (2.14) holds for $M_{0}=(1+M)\left(1-\beta_{0}\right)^{-1}$ and $\omega_{0}=\omega$. Finally, since $x^{(n)}(t)$ are uniformly continuous on $\left[-r, t_{0}\right]$ for every $t_{0}>-r$ and $x^{(n)}(\cdot)$ is continuous for $t \geq 0$ in $\mathcal{X}$, by $(2.18)$, we know that $x_{t}(\cdot)$ is continuous for $t \geq 0$ in $\mathcal{X}$.

3. Robustness with respect to small time-varied delay. In this section we will investigate the stability of the solution of (2.12). To this end, we rewrite $(2.12)$ as

$$
\begin{cases}x^{\prime}(t)=(A+B) x(t)+B(x(t-\tau(t))-x(t)), & t \geq 0, \\ x(\theta)=\xi(\theta), & -r \leq \theta \leq 0,\end{cases}
$$

where $\xi(\cdot) \in \mathcal{X}, 0 \leq \tau(t) \leq r$ and $\tau(t)$ is continuous for $t \geq 0$. The solution of (3.1) is related to the integrated equation

$$
\left\{\begin{array}{rlrl}
x(t)= & T_{B}(t) \xi(0) & \\
& & \\
& +\int_{0}^{t} T_{B}(t-s) B(x(s-\tau(s))-x(s)) d s, & & t \geq 0, \\
x(\theta)= & \xi(\theta), & & -r \leq \theta \leq 0 .
\end{array}\right.
$$

Lemma 3.1. The space $X_{b}$ is $T_{B}(t)$-invariant, i.e., $T_{B}(t) X_{b} \subset X_{b}$ for $t \geq 0$, and $\left(T_{B}(t)\right)_{t \geq 0}$ is a $C_{0}$-semigroup on $X_{b}$. Moreover, if $T_{B}(t)$ is exponentially stable on $X$, then so is $T_{B}(t)$ on $X_{b}$ and

$$
\left\|T_{B}(t) x\right\|_{s} \leq\left(3+k_{1}\left(t_{0}\right)\right) M_{b} e^{\omega_{b} t_{0}} e^{-\omega_{b} t}\|x\|_{s}, \quad t \geq 0, x \in X_{b},
$$

where $t_{0}>0$ is arbitrary, $M_{b}$ and $\omega_{b}$ are positive constants such that $\left\|T_{B}(t)\right\| \leq M_{b} e^{-\omega_{b} t}$ for $t \geq 0$, and $\|\cdot\|_{s}$ is given by (2.11).

Proof. It is easy to see that $X_{b}$ is $T_{B}(t)$-invariant. Now we suppose that $T_{B}(t)$ is exponentially stable on $X$. Let $x \in X_{b}$ and $t_{0}>0$. Then for every $\varepsilon>0$, there is a $T_{\varepsilon} \geq t_{0}$ such that for $s \geq T_{\varepsilon}$ and $t \geq 0$,

$$
\begin{aligned}
\left\|B T_{B}(t+s) x-B T_{B}(s) x\right\| & =\left\|B T_{B}\left(t_{0}\right)\left(T_{B}\left(t+s-t_{0}\right) x-T_{B}\left(s-t_{0}\right) x\right)\right\| \\
& \leq k_{1}\left(t_{0}\right) M_{b}\left(e^{-\omega_{b}\left(t+s-t_{0}\right)}+e^{-\omega_{b}\left(s-t_{0}\right)}\right)\|x\|<\varepsilon / 2 .
\end{aligned}
$$

On the other hand, by Lemma $2.3, B T_{B}(s) x$ is continuous for $s \geq 0$, and therefore uniformly continuous on $\left[0, T_{\varepsilon}+1\right]$. So we can find $\delta_{\varepsilon} \in(0,1)$ such that when $t \in\left[0, \delta_{\varepsilon}\right]$,

$$
\left\|B T_{B}(t+s) x-B T_{B}(s) x\right\|<\varepsilon / 2, \quad s \in\left[0, T_{\varepsilon}\right] .
$$


Therefore, for $t \in\left[0, \delta_{\varepsilon}\right]$, we have

$$
\begin{aligned}
\left\|T_{B}(t) x-x\right\|_{s} & =\left\|T_{B}(t) x-x\right\|+\sup _{s \geq 0}\left\|B T_{B}(s)\left(T_{B}(t) x-x\right)\right\| \\
& \leq \varepsilon / 2+\varepsilon / 2=\varepsilon,
\end{aligned}
$$

which proves the strong continuity of $T_{B}(t)$ on $\left(X_{b},\|\cdot\|_{s}\right)$.

Next we show that $T_{B}(t)$ is exponentially stable on $\left(X_{b},\|\cdot\|_{s}\right)$ and (3.3) holds. In fact, for $x \in X_{b}$ and $t \geq t_{0}>0$, we have

$$
\begin{aligned}
\left\|T_{B}(t) x\right\|_{s} & =\left\|T_{B}(t) x\right\|+\sup _{s \geq 0}\left\|B T_{B}(s) T_{B}(t) x\right\| \\
& =\left\|T_{B}(t) x\right\|+\sup _{s \geq 0}\left\|B T_{B}(s+t) x\right\| \\
& =\left\|T_{B}(t) x\right\|+\sup _{s \geq 0}\left\|B T_{B}\left(t_{0}\right) T_{B}\left(t+s-t_{0}\right) x\right\| \\
& \leq M_{b} e^{-\omega_{b} t}\|x\|+\sup _{s \geq 0} k_{1}\left(t_{0}\right) M_{b} e^{-\omega_{b}\left(t+s-t_{0}\right)}\|x\| \\
& \leq\left(1+k_{1}\left(t_{0}\right) e^{\omega_{b} t_{0}}\right) M_{b} e^{-\omega_{b} t}\|x\|_{s},
\end{aligned}
$$

and for $0 \leq t \leq t_{0}$,

$$
\begin{aligned}
\left\|T_{B}(t) x\right\|_{s} & =\left\|T_{B}(t) x\right\|+\sup _{s \geq 0}\left\|B T_{B}(s+t) x\right\| \\
& =M_{b} e^{-\omega_{b} t}\|x\|+\|x\|_{s} \leq\left(M_{b}+e^{\omega_{b} t_{0}}\right) e^{-\omega_{b} t}\|x\|_{s} .
\end{aligned}
$$

This implies (3.3) since $M_{b}, e^{\omega_{b} t_{0}} \geq 1$.

In the following we will assume that $T_{B}(t)$ is exponentially stable on $X$, and adopt the $\|\cdot\|_{s}$-norm on $X_{b}$. Note that by Lemma 2.4 , this norm is equivalent to the $\|\cdot\|_{b}$-norm.

Definition 3.2. We say that the exponential stability of $T_{B}(t)$ with small time-varied delay on the phase space $\mathcal{X}$ is robust or the solutions of (3.2) in $\mathcal{X}$ are uniformly exponentially stable with small time-varied delay if there are positive constants $r_{0}, M_{0}$, and $\omega_{0}$ such that for $t \geq 0,0 \leq \tau(t) \leq$ $r \leq r_{0}$ continuous and $\xi \in \mathcal{X}$,

$$
\left\|T_{r}(t) \xi\right\|_{\mathcal{X}} \leq M_{0} e^{-\omega_{0} t}\|\xi\|_{\mathcal{X}}
$$

REMARK 3.3. The robustness defined above has some kind of uniformity since the constants $M_{0}$ and $\omega_{0}$ (depend on $r_{0}$ ) are independent of $r$.

Our main result is

THEOREM 3.4. If $B T(t)$ is norm continuous for $t>0$, then the exponential stability of $T_{B}(t)$ with small time-varied delay on the phase space $\mathcal{X}$ is robust. 
Proof. Suppose that $\left\|T_{B}(t)\right\| \leq M_{b} e^{-\omega_{b} t}$ for $t \geq 0$. By Lemma 3.1, $T_{B}(t)$ is exponentially stable on $X_{b}$ and (3.3) holds. Since $B T(t)$ is norm continuous for $t>0$, so is $B T_{B}(t)$ by Lemma 2.2. For $r>0$ and $\xi \in \mathcal{X}$, by Theorem $2.5,(3.2)$ has a unique solution $x_{t}(\cdot)=x_{t}(\cdot, \xi)=x(t+\cdot, \xi) \in \mathcal{X}$ and

$$
\left\|x_{t}(\cdot)\right\|_{\mathcal{X}} \leq N_{0} e^{\sigma_{0} t}\|\xi\|_{\mathcal{X}}
$$

where $N_{0}$ and $\sigma_{0}$ are independent of $r$. For $\omega_{1} \in\left(0, \omega_{b}\right)$ and $t_{0}>0$, note that

$$
\begin{aligned}
e^{\omega_{1} t} k_{1}(t) & =e^{\omega_{1} t}\left\|B T_{B}(t)\right\|=e^{\omega_{1} t}\left\|B T_{B}\left(t_{0}\right) T_{B}\left(t-t_{0}\right)\right\| \\
& \leq k_{1}\left(t_{0}\right) M_{b} e^{\omega_{1} t} e^{-\omega_{b}\left(t-t_{0}\right)} .
\end{aligned}
$$

For $t \geq t_{0}$ and $k_{1} \in L_{\mathrm{loc}}^{1}(0, \infty)$, we have

$$
\begin{aligned}
\beta_{2} & :=\int_{0}^{\infty} e^{\omega_{1} t} k_{1}(t) d t<\infty \\
\eta(t) & :=\sup _{s \geq 0} \int_{s}^{s+t} e^{\omega_{1} \tau} k_{1}(\tau) d \tau \rightarrow 0 \quad \text { as } t \rightarrow 0+.
\end{aligned}
$$

Choose $\tau_{0} \in(0,1]$ small enough such that

$$
\begin{aligned}
& \left(e^{\omega_{b}}+1\right) \eta\left(\tau_{0}\right)<1 \\
& e^{\omega_{b}}\left[\tau_{0} M_{b}\left(\frac{1}{M_{1}}+\frac{1}{\omega_{b}-\omega_{1}}\right)+2 \eta\left(\tau_{0}\right)\right]\left(1-\left(e^{\omega_{b}}+1\right) \eta\left(\tau_{0}\right)\right)^{-1}<1 .
\end{aligned}
$$

Since $B T_{B}(t)$ is norm continuous for $t>0$, for $r_{1}=t_{0} / 2$ there exists $r_{0} \in$ $\left(0, r_{1}\right)$ such that

$$
\left\|B T_{B}\left(r_{1}-r\right)-B T_{B}\left(r_{1}\right)\right\|<r_{1}, \quad 0 \leq r \leq r_{0} .
$$

Now we estimate $\|B x(t-\tau(t))-B x(t)\|$ for $t \geq 0$, where $0 \leq \tau(t) \leq r \leq r_{0}$ and $\tau(t)$ is continuous for $t \geq 0$. For $t \in\left[0, \tau_{0}\right]$, since $\tau_{0} \leq 1$, by (3.4) we have

$$
\begin{aligned}
\|B x(t-\tau(t))-B x(t)\| & \leq\|x(t-\tau(t))-x(t)\|_{s} \leq 2\left\|x_{t}(\cdot)\right\|_{\mathcal{X}} \\
& \leq 2 N_{0} e^{\sigma_{0} t}\|\xi\|_{\mathcal{X}} \leq 2 N_{0} e^{\sigma_{0}}\|\xi\|_{\mathcal{X}} .
\end{aligned}
$$

Let $M_{1}=2 N_{0} e^{2 \sigma_{0}}$. We will prove that

$$
\|B x(t-\tau(t))-B x(t)\| \leq M_{1} e^{\omega_{1} t}\|\xi\|_{\mathcal{X}}, \quad t \geq 0, \xi \in \mathcal{X} .
$$

For $t \in\left[0, \tau_{0}\right]$, we know from (3.7) that (3.8) holds. Next, suppose that (3.8) holds for $t \in\left[0, n \tau_{0}\right]$, where $n$ is any positive integer, and let $t \in$ $\left[n \tau_{0},(n+1) \tau_{0}\right]$. If $t-\tau(t)>n \tau_{0}$, then by (3.6) and (3.8), we have 


$$
\begin{aligned}
& \|B x(t-\tau(t))-B x(t)\| \\
& =\| B\left(T_{B}(t-\tau(t))-T_{B}(t)\right) \xi(0) \\
& +\int_{0}^{t-\tau(t)} B T_{B}(t-\tau(t)-s) B(x(s-\tau(s))-x(s)) d s \\
& -\int_{0}^{t} B T_{B}(t-s) B(x(s-\tau(s))-x(s)) d s \| \\
& \leq\left\|B\left(T_{B}\left(r_{1}-\tau(t)\right)-T_{B}\left(r_{1}\right)\right) T_{B}\left(t-r_{1}\right) \xi(0)\right\| \\
& +\int_{0}^{n \tau_{0}-r_{1}}\left\|B\left(T_{B}\left(r_{1}-\tau(t)\right)-T_{B}\left(r_{1}\right)\right)\right\| \\
& \cdot\left\|T_{B}\left(t-r_{1}-s\right)\right\| \cdot\|B(x(s-\tau(s))-x(s))\| d s \\
& +\int_{n \tau_{0}-r_{1}}^{n \tau_{0}}\left[k_{1}(t-\tau(t)-s)+k_{1}(t-s)\right]\|B(x(s-\tau(s))-x(s))\| d s \\
& t-\tau(t) \\
& +\int_{n \tau_{0}} k_{1}(t-\tau(t)-s)\|B(x(s-\tau(s))-x(s))\| d s \\
& +\int_{n \tau_{0}}^{t} k_{1}(t-s)\|B(x(s-\tau(s))-x(s))\| d s,
\end{aligned}
$$

and the first three terms on the right-hand side are bounded by

$$
\begin{aligned}
r_{1} M_{b} e^{-\omega_{b}\left(t-r_{1}\right)}\|\xi\| \mathcal{X}+\int_{0}^{n \tau_{0}-r_{1}} r_{1} M_{b} e^{-\omega_{b}\left(t-r_{1}\right)}\|\xi\| \mathcal{X} d s \\
+\int_{n \tau_{0}-r_{1}}^{n \tau_{0}}\left[k_{1}(t-\tau(t)-s)+k_{1}(t-s)\right] M_{1} e^{-\omega_{1} s}\|\xi\|_{\mathcal{X}} d s \\
\leq r_{1} M_{b} e^{-\omega_{b}\left(t-r_{1}\right)}\|\xi\|_{\mathcal{X}}+r_{1} M_{b} M_{1} \int_{t-n \tau_{0}}^{t-r_{1}} e^{-\omega_{b} \tau} e^{-\omega_{1}\left(t-r_{1}-\tau\right)} d \tau\|\xi\|_{\mathcal{X}} \\
+\int^{t-n \tau_{0}+r_{1}-\tau(t)} k_{1}(\tau) e^{-\omega_{1}(t-\tau(t)-\tau)} d \tau \\
\left.\quad+\int_{t-n \tau_{0}+r_{1}}^{t-n \tau_{0}-\tau(t)} k_{1}(\tau) e^{-\omega_{1}(t-\tau)} d \tau\right] M_{1}\|\xi\|_{\mathcal{X}} \\
\leq\left[r_{1} M_{b} e^{\omega_{b}}\left(1+M_{1}\left(\omega_{b}-\omega_{1}\right)^{-1}\right)+2 M_{1} e^{\omega_{b}} \eta\left(r_{1}\right)\right] e^{-\omega_{1} t}\|\xi\|_{\mathcal{X}} \\
\leq M_{2} e^{-\omega_{1} t}\|\xi\| \mathcal{X},
\end{aligned}
$$


where $M_{2}:=M_{1} \varrho, \varrho:=e^{\omega_{b}}\left[\tau_{0} M_{b}\left(M_{1}^{-1}+\left(\omega_{b}-\omega_{1}\right)^{-1}\right)+2 \eta\left(\tau_{0}\right)\right]$. Therefore, $(3.9)$

$$
\begin{aligned}
& \|B x(t-\tau(t))-B x(t)\| \\
& \leq M_{2} e^{-\omega_{1} t}\|\xi\| \mathcal{X}+\int_{n \tau_{0}}^{t-\tau(t)} k_{1}(t-\tau(t)-s)\|B(x(s-\tau(s))-x(s))\| d s \\
& \quad+\int_{n \tau_{0}}^{t} k_{1}(t-s)\|B(x(s-\tau(s))-x(s))\| d s .
\end{aligned}
$$

Then by the generalized Gronwall inequality or by induction, from (3.9), we have for $t-\tau(t)>n \tau_{0}, t \in\left[n \tau_{0},(n+1) \tau_{0}\right]$,

$$
\|B(x(t-\tau(t))-x(t))\| \leq \sum_{n=0}^{\infty} y^{(n)}(t),
$$

where $y^{(0)}(t):=M_{2} e^{-\omega_{1} t}\|\xi\|_{\mathcal{X}}$ and for $n=1,2, \ldots$,

$$
y^{(n)}(t):=\int_{n \tau_{0}}^{t-\tau(t)} k_{1}(t-\tau(t)-s) y^{(n-1)}(s) d s+\int_{n \tau_{0}}^{t} k_{1}(t-s) y^{(n-1)}(s) d s .
$$

Hence,

$$
\begin{aligned}
y^{(1)}(t)= & \int_{n \tau_{0}}^{t-\tau(t)} k_{1}(t-\tau(t)-s) y^{(0)}(s) d s+\int_{n \tau_{0}}^{t} k_{1}(t-s) y^{(0)}(s) d s \\
= & {\left[\int_{0}^{t-n \tau_{0}-\tau(t)} k_{1}(\tau) e^{-\omega_{1}(t-\tau(t)-\tau)} d \tau\right.} \\
& \left.+\int_{0}^{t-n \tau_{0}} k_{1}(\tau) e^{-\omega_{1}(t-\tau)} d \tau\right] M_{2}\|\xi\|_{\mathcal{X}} \\
\leq & M_{2}\left(e^{\omega_{1} r}+1\right) \eta\left(\tau_{0}\right) e^{-\omega_{1} t}\|\xi\|_{\mathcal{X}}
\end{aligned}
$$

and then by induction,

$$
y^{(n)}(t) \leq M_{2}\left(e^{\omega_{1} r}+1\right)^{n} \eta\left(\tau_{0}\right)^{n} e^{-\omega_{1} t}\|\xi\|_{\mathcal{X}}, \quad n=0,1,2, \ldots
$$

Now (3.10) and (3.11) imply

$$
\begin{aligned}
\|B(x(t-\tau(t))-x(t))\| & \leq \sum_{n=0}^{\infty} M_{2}\left(e^{\omega_{1} r}+1\right)^{n} \eta\left(\tau_{0}\right)^{n} e^{-\omega_{1} t}\|\xi\|_{\mathcal{X}} \\
& =M_{2}\left[1-\left(e^{\omega_{1} r}+1\right) \eta\left(\tau_{0}\right)\right]^{-1} e^{-\omega_{1} t}\|\xi\|_{\mathcal{X}} \\
& \leq M_{2}\left[1-\left(e^{\omega_{b}}+1\right) \eta\left(\tau_{0}\right)\right]^{-1} e^{-\omega_{1} t}\|\xi\|_{\mathcal{X}}
\end{aligned}
$$


Note that by (3.5), $M_{2}\left[1-\left(e^{\omega_{b}}+1\right) \eta\left(\tau_{0}\right)\right]^{-1}<M_{1}$, and thus (3.8) holds for $t \in\left[n \tau_{0},(n+1) \tau_{0}\right]$ and $t-\tau(t) \geq n \tau_{0}$. But from the calculations above it is easy to see that (3.12) is also valid for $t \in\left[n \tau_{0},(n+1) \tau_{0}\right]$ with $t-\tau(t) \leq n \tau_{0}$. Therefore, (3.8) holds for all $t \geq 0$.

Finally, we estimate $\left\|x_{t}(\cdot)\right\|_{\mathcal{X}}$. For $t \geq \tau_{0},-r \leq \theta \leq 0$ and $0 \leq \tau(t) \leq$ $r \leq r_{0}$, by (3.3) and (3.8) we have

$$
\begin{aligned}
\left\|x_{t}(\theta)\right\|_{s}= & \left\|T_{B}(t+\theta) \xi(0)+\int_{0}^{t+\theta} T_{B}(t+\theta-s) B(x(s-\tau(s))-x(s)) d s\right\|_{s} \\
\leq & \left\|T_{B}(t+\theta) \xi(0)\right\|_{s}+\left\|\int_{0}^{t+\theta} T_{B}(t+\theta-s) B(x(s-\tau(s))-x(s)) d s\right\|_{s} \\
\leq & \left(3+k_{1}(1)\right) e^{\omega_{b}} M_{b} e^{-\omega_{b} t}\|\xi(0)\|_{s} \\
& +\left\|\int_{0}^{t+\theta} T_{B}(t+\theta-s) B(x(s-\tau(s))-x(s)) d s\right\| \\
& +\sup _{\sigma \geq 0}\left\|\int_{0}^{t+\theta} B T_{B}(t+\theta-s) T_{B}(\sigma) B(x(s-\tau(s))-x(s)) d s\right\| \\
\leq & \left(3+k_{1}(1)\right) e^{\omega_{b}} M_{b} e^{-\omega_{b} t}\|\xi\|_{\mathcal{X}} \\
& +\int_{0}^{t+\theta} M_{b} e^{-\omega_{b}(t+\theta-s)} M_{1} e^{-\omega_{1} s} M_{1} e^{-\omega_{1} s}\|\xi\|_{\mathcal{X}} d s \\
& +M_{b} \int_{0}^{t+\theta} k_{1}(t+\theta-s) M_{1} e^{-\omega_{1} s}\|\xi\|_{\mathcal{X}} d s \\
\leq & M_{b} M_{1} e^{\omega_{b}}\left[\left(3+k_{1}(1)\right) M_{1}^{-1}+\left(\omega_{b}-\omega_{1}\right)^{-1}+\beta_{2}\right] e^{-\omega_{1} t}\|\xi\|_{\mathcal{X}}
\end{aligned}
$$

which proves

$$
\left\|x_{t}(\cdot)\right\|_{\mathcal{X}} \leq M_{3} e^{-\omega_{1} t}\|\xi\|_{\mathcal{X}}, \quad t \geq \tau_{0}, r \leq r_{0},
$$

where $M_{3}:=M_{b} M_{1} e^{\omega_{b}}\left[\left(3+k_{1}(1)\right) M_{1}^{-1}+\left(\omega_{b}-\omega_{1}\right)^{-1}+\beta_{2}\right]$. Moreover, for $t \in\left[0, \tau_{0}\right]$, by $(3.4)$, we have

$$
\begin{aligned}
\left\|x_{t}(\cdot)\right\|_{\mathcal{X}} & \leq N_{0} e^{\sigma_{0} \tau_{0}}\|\xi\|_{\mathcal{X}} \leq N_{0} e^{\sigma_{0} \tau_{0}} e^{\omega_{1} \tau_{0}} e^{-\omega_{1} t}\|\xi\|_{\mathcal{X}} \\
& \leq N_{0} e^{\sigma_{0}+\omega_{b}} e^{-\omega_{1} t}\|\xi\|_{\mathcal{X}} .
\end{aligned}
$$

Therefore, for $t \geq 0, r \in\left[0, r_{0}\right]$, and $\xi \in \mathcal{X}$,

$$
\left\|x_{t}(\cdot)\right\|_{\mathcal{X}} \leq\left(M_{3}+N_{0} e^{\sigma_{0}+\omega_{b}}\right) e^{-\omega_{1} t}\|\xi\|_{\mathcal{X}}
$$

EXAMPLE 3.5. Let $H_{1}, H_{2}$ be Hilbert spaces. Suppose that $A_{j}$ generates a $C_{0}$-semigroup $T_{j}(t)$ on $H_{j}$ for $j=1,2$ respectively, and $T_{2}(\cdot)$ is holomor- 
phic. Moreover, suppose that $B_{1}: D\left(B_{1}\right) \subset H_{1} \rightarrow H_{2}$ is a closed linear operator satisfying $D\left(B_{1}\right) \supset D\left(\left(-A_{2}\right)^{r}\right)$, where $0<r<1$. Since $T_{2}(t)$ is holomorphic, by $[\mathrm{EN}], B_{1} T_{2}(t) \in \mathbf{B}\left(H_{2}, H_{1}\right)$ and there exist constants $M$ and $\omega$ such that $\left\|B_{1} T_{2}(t)\right\| \leq M e^{\omega t} / t^{r}=: k(t)$ for $t>0$. Let $H=H_{1} \times H_{2}$,

$$
A=\left(\begin{array}{cc}
A_{1} & 0 \\
0 & A_{2}
\end{array}\right), \quad B=\left(\begin{array}{cc}
0 & B_{1} \\
0 & 0
\end{array}\right) .
$$

Then $A$ generates a $C_{0}$-semigroup

$$
T(t)=\left(\begin{array}{cc}
T_{1}(t) & 0 \\
0 & T_{2}(t)
\end{array}\right), \quad t \geq 0
$$

on $H$ and

$$
B T(t)=\left(\begin{array}{cc}
0 & B_{1} \\
0 & 0
\end{array}\right)=\left(\begin{array}{cc}
T_{1}(t) & 0 \\
0 & T_{2}(t)
\end{array}\right)=\left(\begin{array}{cc}
0 & B_{1} T_{2}(t) \\
0 & 0
\end{array}\right)
$$

is norm continuous for $t>0$ with $\|B T(t)\|=\left\|B_{1} T_{2}(t)\right\|_{\mathbf{B}\left(H_{2}, H_{1}\right)} \leq k(t) \in$ $L_{\text {loc }}^{1}(0, \infty)$. So the operators $A$ and $B$ satisfy the assumptions of Theorems 2.5 and 3.4 , but the $C_{0}$-semigroup $T(t)$ is not holomorphic.

Acknowledgements. The authors are greatly indebted to the referees for helpful suggestions and for pointing out a mistake in the proof of Theorem 2.5.

\section{References}

[Ba1] A. Bátkai, On the stability of linear partial differential equations with delay, Tübinger Berichte Funktionalanalysis 9 (1999/2000), 47-56.

[Ba2] A. Bátkai and S. Piazzera, Semigroups and linear partial differential equations with delay, J. Math. Anal. Appl. 264 (2001), 1-20.

[Da] R. Datko, Not all feedback stabilized hyperbolic systems are robust with respect to small time delays in their feedbacks, SIAM J. Control Optim. 26 (1988), 697-713.

[DS] N. Dunford and J. Schwartz, Linear Operators, Part I: General Theory, Interscience, 1958.

[EN] K. J. Engel and R. Nagel, One-Parameter Semigroups for Linear Evolution Equations, Grad. Texts in Math. 194, Springer, 1999.

[FN] A. Fisher and J. M. A. M. van Neerven, Robust stability of $C_{0}$-semigroups and applications to stability of delay equations, J. Math. Anal. Appl. 226 (1998), 82100.

[Hu] F. L. Huang, On the problem of stability of solutions of linear differential equations with small delay in Banach spaces, J. Math. (Wuhan) 6 (1986), 183-192 (in Chinese).

[JGH] W. S. Jiang, F. M. Guo and F. L. Huang, Robustness with respect to small delays for exponential stability of linear dynamical systems with unbounded operator in the delay term, preprint. 
[Liu] K. S. Liu, Differentiability of infinite-dimensional linear systems with time delay and its applications to stability analysis, J. Systems Sci. Math. Sci. 12 (1992), 297-306 (in Chinese).

Department of Mathematics

Sichuan University

Chengdu, Sichuan 610064, P.R. China

E-mail: limiao1973@hotmail.com

xiaohuigu@sohu.com

Received May 8, 2003

Revised version December 18, 2003 